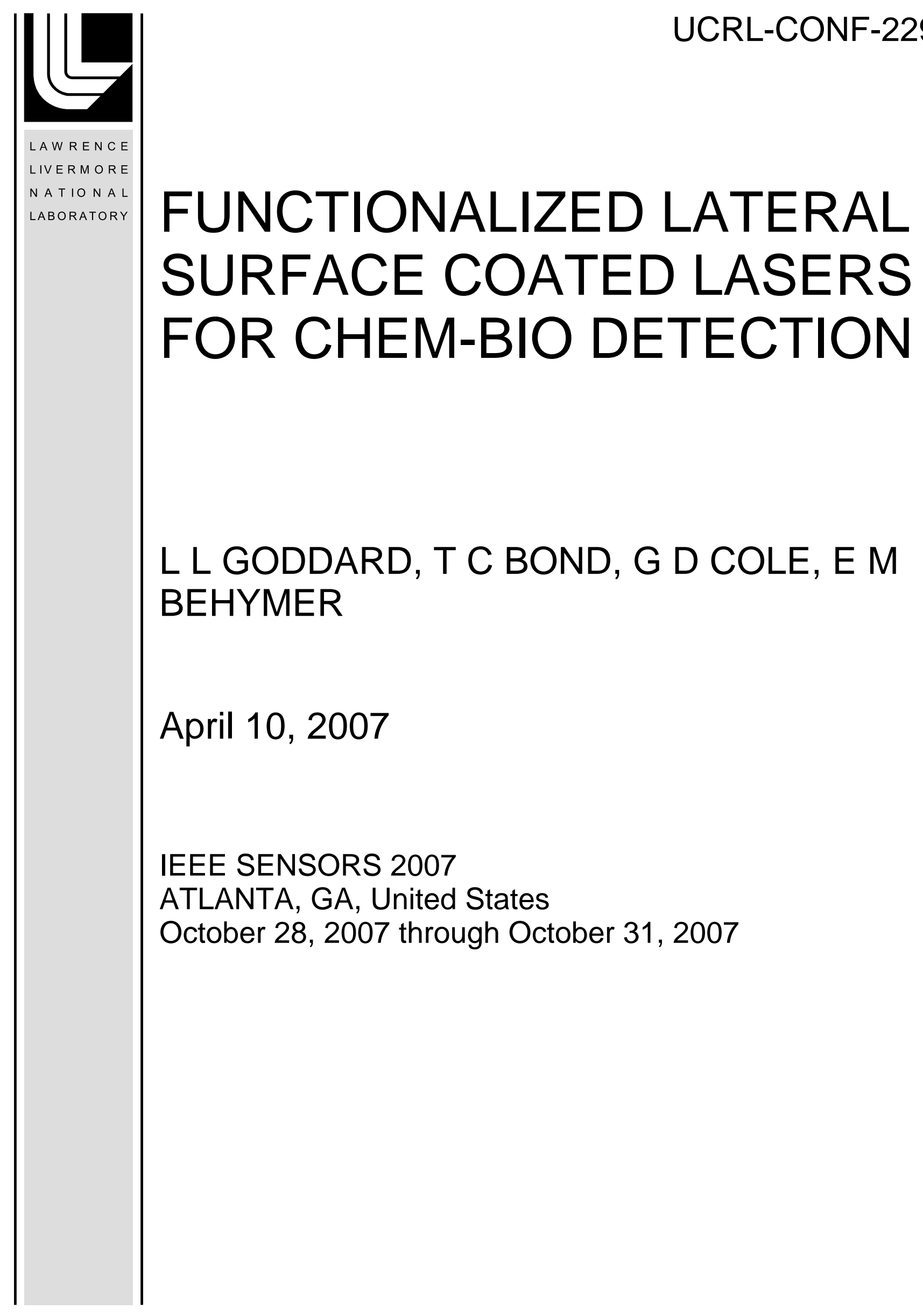


This document was prepared as an account of work sponsored by an agency of the United States Government. Neither the United States Government nor the University of California nor any of their employees, makes any warranty, express or implied, or assumes any legal liability or responsibility for the accuracy, completeness, or usefulness of any information, apparatus, product, or process disclosed, or represents that its use would not infringe privately owned rights. Reference herein to any specific commercial product, process, or service by trade name, trademark, manufacturer, or otherwise, does not necessarily constitute or imply its endorsement, recommendation, or favoring by the United States Government or the University of California. The views and opinions of authors expressed herein do not necessarily state or reflect those of the United States Government or the University of California, and shall not be used for advertising or product endorsement purposes. 


\section{Functionalized Lateral Surface Coated Lasers for Chem-Bio Detection}

Lynford L. Goddard, Tiziana C. Bond, Garrett D. Cole, and Elaine M. Behymer

Lawrence Livermore National Laboratory, Livermore, CA, 94550, goddard5@1lnl.gov

\section{Summary}

We present a class of compact, monolithic, photonic sensors that consist of multiple section edge emitting lasers (EELs) with lateral surface coatings that are functionalized to detect low levels of chemical or biological stimulants. Specifically, we discuss a $10 \mu \mathrm{m} \times 1000 \mu \mathrm{m}_{2}$ sensor with a Pd coating and techniques to enhance the sensitivity by $35 \mathrm{x}$ and reduce the minimum detection limit (MDL) to $2 \mathrm{ppm}$. Compared to conventional optical $\mathrm{H}_{2}$ sensors that use fiber gratings, surface plasmon resonances, or surface reflectance, our sensors offer the advantages of smaller size, monolithic integration of laser source and detector, and 1-D scalability to an array of sensors that are functionalized to detect different agents.

\section{Motivation}

Our goal is to develop chip-scale sensor networks to detect and analyze unknown chemical and biological agents in-situ. Traditional optical sensors ${ }^{1,2,3}$ can be difficult to integrate or array. Fig. 1 shows the layout for our generic class of enhanced sensors. The sensor is a multiple section $5 \mu \mathrm{m} \times 1000 \mu \mathrm{m}$ EEL with a surface coating that extends $2.5 \mu \mathrm{m}$ laterally on either side. The waveguide mode has a small overlap, $\sim 10^{-4}$, with the thin coating. The coating's optical properties (e.g. extinction coefficient) change when molecules adsorb to the surface. For example, $\mathrm{H}_{2}$ gas changes $\mathrm{Pd}$ to $\mathrm{PdH}$ and reduces the internal loss, $\alpha_{\mathrm{i}}$, from $57 \mathrm{~cm}^{-1}$ at a rate of $1.5 \mathrm{~cm}^{-1}$ per percent $\mathrm{H}_{2}$. Thus, the laser's output power varies in response to the concentration of adsorbed $\mathrm{H}_{2}$. To maximize sensitivity, the laser is operated at the threshold knee. The $\mathrm{MDL}$ is determined by assuming the minimum easily measurable fractional power change, $\Delta \mathrm{P} / \mathrm{P}$, is $10^{-3}$.

\section{Results}

A 1-D rate equation model, which was verified previously against experimental L-I data, was used to calculate the sensor response (see Fig. 2) for a Pd coated passive waveguide, a single section EEL, and a multiple section EEL that has a $50 \mu \mathrm{m}$ saturable absorber as the center section of Fig. 1. The saturable absorber amplifies the nonlinearity of the lasing knee according to the gain lever effect. The MDL of the three structures is predicted to be $70 \mathrm{ppm}, 12 \mathrm{ppm}$, and 2ppm, respectively. Fig. 3 shows another class of generic sensors for higher sensitivity threshold detection. The multiple section EEL with lateral coatings is embedded inside slightly detuned DBR mirrors. As before $\mathrm{H}_{2}$ reduces $\alpha_{i}$ and increases the circulating power, $\mathrm{P}_{\text {circ, }}$ which reduces the average carrier density, $\mathrm{N}_{\text {avg }}$, due to increased stimulated emission. This changes the round-trip cavity phase. The phase shift is enhanced in multiple section lasers according to the gain-index lever ${ }^{4}$ (see Fig. 4a). The cavity length is chosen such that the spacing of the wavelengths satisfying the round-trip phase condition is $1-2 \%$ smaller than the spacing of the gain peaks from the detuned DBR mirrors. This creates a Vernier effect (see Fig. 4b) which magnifies the phase shift ${ }^{4}$ caused by the presence of $\mathrm{H}_{2}$. When the $\mathrm{H}_{2}$ concentration increases above a controllable threshold, the laser hops from $\lambda_{1}$ to $\lambda_{2}$. The specially designed DBR mirrors ${ }^{4}$ (see Fig. 5) cause the highly reflective (HR) facet and antireflective (AR) facet to swap as the wavelength changes from $\lambda_{1}$ to $\lambda_{2}$. Thus, above the given $\mathrm{H}_{2}$ threshold, the output from the left facet turns on. Course electronic control of this $\mathrm{H}_{2}$ threshold level is possible by adjusting the biases on the slave and control sections since they determine the strength of the gain-index lever. Fine electronic control can be achieved by adjusting the bias on the phase section. If the phase in the absence of $\mathrm{H}_{2}$ is set near the mode hop point, an extremely low MDL can be achieved, limited only by the stability of the drive currents and ambient environmental conditions.

\footnotetext{
${ }^{1}$ P. Tobiska et. al, “An integrated optic hydrogen sensor based on SPR on palladium,” Sens Act B, p. 168 (2001).

${ }^{2}$ C. Christofides and A. Mandelis, "Solid-state sensors for trace hydrogen detection," J. Appl. Phys., p. R1 (1990).

${ }^{3}$ A. Mandelis and J. Garcia, "Pd/PVDF thin film hydrogen sensor based on laser...," Sens Act B, p 258 (1998).

${ }^{4}$ L. Goddard et. al, "Rapidly reconfigurable all-optical universal logic gates," Proc. SPIE, pp. 63680H-1-13 (2006).
} 


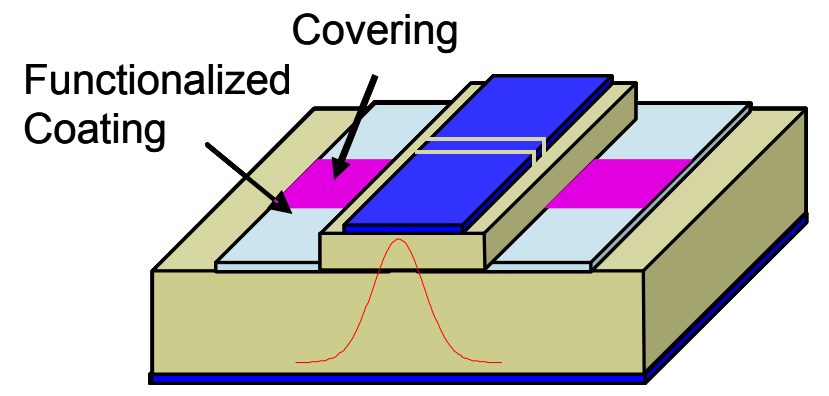

Fig. 1: Multiple section laser with Pd coating for $\mathrm{H}_{2}$ sensing. A sensor array with various cover lengths enables compensation for system drift or temperature.

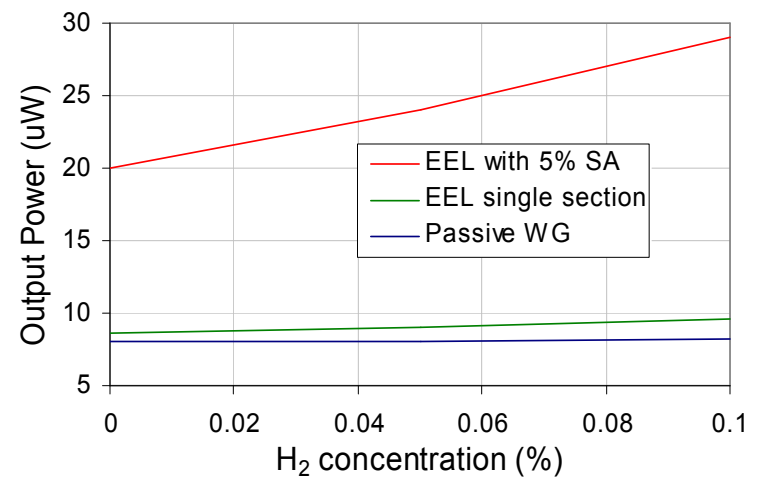

Fig. 2: Simulated sensor responses for Pd coated passive WG, single section EEL, and multi-section EEL (5\% saturable absorber). $\mathrm{H}_{2}+\mathrm{Pd} \rightarrow \mathrm{PdH}$, reduces $\alpha_{\mathrm{i}}$ and increases $\mathrm{P}_{\text {out }}$.

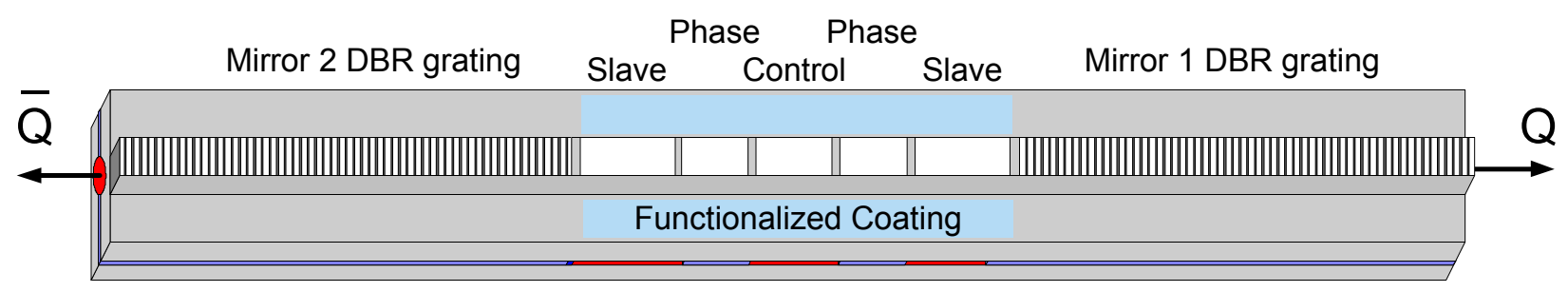

Fig. 3: Functionalized enhanced photonic sensor with threshold detection. Here, as $\mathrm{H}_{2}$ reduces $\mathrm{a}_{\mathrm{i}}, \mathrm{P}_{\text {circ }}$ increases and $\mathrm{N}_{\text {avg }}$ decreases. The resulting cavity phase shift is enhanced by the gain-index lever and

Vernier effects and causes the laser to hop to the mode that outputs light on the opposite side.
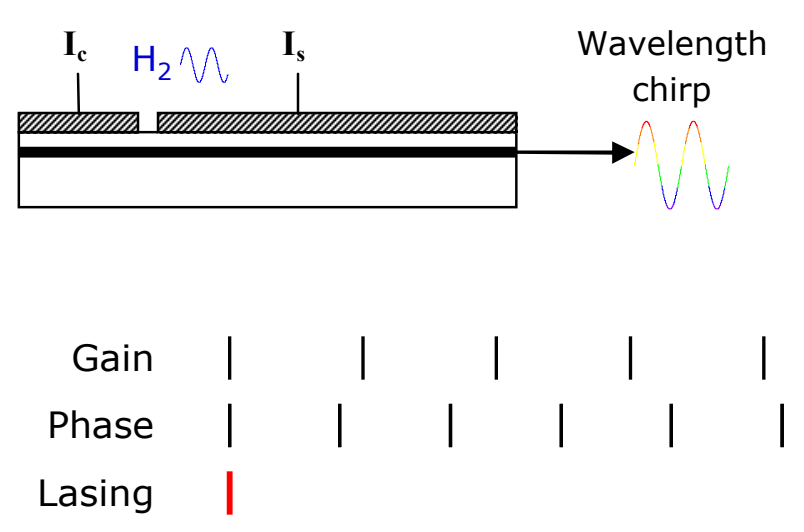

Fig. 4: (a) The gain-index lever in multiple section lasers is used to enhance the phase shift caused by the decrease in $\mathrm{N}_{\text {avg }}$ due to $\mathrm{H}_{2}$. (b) The next mode is aligned using the Vernier effect, which magnifies the shift.

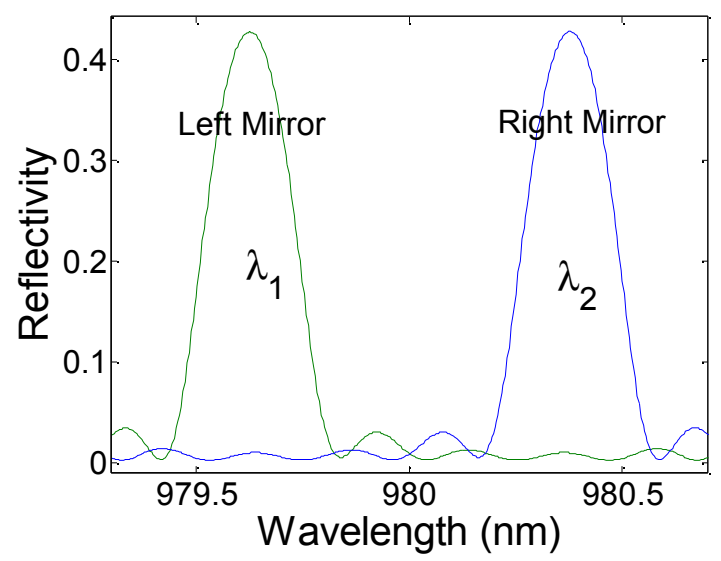

Fig. 5: Desired reflectivity profile for an alternating facet laser. At $\lambda_{1}$, the left mirror is HR and the right is AR so laser light exits the right facet. $\mathrm{H}_{2}$ causes the mode to hop to $\lambda_{2}$ and switches the light to the left facet.

This work was performed under the auspices of the U. S. Department of Energy (DOE) by the University of California, Lawrence Livermore National Laboratory (LLNL) under Contract No. W 7405 Eng 48. 